\title{
KÜMMELL ON RESECTION OF THE KIDNEY.'
}

AFTER experimenting on animals by removing pieces of varying size from normal kidneys, Kümmell learned that the operation could be done without danger. He has since had opportunities to practice upon the human subject the resection of diseased kidney tissue, a report of which operations he made before the last German Surgical Congress.

The first -case upon which he operated was a woman aged fortyone years. She began to complain of pain in the abdomen and loin. There was never any pain on urinating. She grew weak and lost appetite, and finally sought medical aid. The physician found a painless turnor the size of a fist just below the right costal arch. At this time, aside from the general weakness, pallor and pain in the loin, and especially in the right side, she complained of nothing. It could not be decided to what organ the tumor belonged until the urine had been examined. At times the urine contained a considerable amount of purulent sediment, and at other times it was clear and normal. The reaction was acid. A small amount of albumin was occasionally present. There was no cystitis. The patient had never passed any gravel. Nine months after the onset of the symptoms, the tumor was exposed by an incision parallel to the free border of the ribs. A fluctuating tumor springing from the upper third of the kidney presented. After separating a few adhesions, the half of the enlarged organ was brought out, and the finctuating part freely. opened. A large amount of pus escaped. The finger introduced into the abscess came against a stone moulded in the shape of the pelvis, which was removed with great difficulty. From the pelvis pus had forced an opening back through the parenchyma and formed a large abscess cavity in the substance of the organ. The walls of this carity were cut away with the scissors, and the cavity partially closed with sutures. The hremorrhage could not be completely checked, so the kidney was sutured in the abdominal wound and tamponed with iodoform gauze. More than a third of the entire organ was removed.

1 Verhandlungen der deutschen Gesellschaft für Chirurgie, xxı Kongress, I $\mathrm{S}_{93}$. 
The wound healed well. At no time did a drop of urine escape from the wound. The urine contained much pus inmediately after the operation. This steadily diminished till, after eight days, it was normal. After a month she returned home with a small granulating wound. Just before this wound closed a small bit of gravel escaped. Three years later she is still perfectly healthy.

The second case reported was that of a man fifty-four years old, who presented himself in a condition of cachexia and complaining of pain in the region of the right kidney. The urine was strongly. stained with blood, cloudy and albuminous. Examination under an anasthetic disclosed nothing abnormal about the kidneys, and examination of the bladder with the soft and hard catheter was negative. Washing the bladder had no influence on the condition of the urine. Cystoscopic examination was negative. The right kidney was possibly slightly larger than the left. This kidney-region was always very painful and tender. A diagnosis of malignant disease of the kidney was made, and the organ exposed by an incision parallel to the costal arch. The kidney was not especially firmly fixed in its bed, and was easily drawn forward. The microscopic appearances of the organ were normal as to consistency, size and color. At the !lyper end was discorered an area which was anamic and dense and sharply outlined from the rest of the organ. It was abont the size of a walnut. This was regarded as malignant, and the whole mass excised in the shape of a wedge, the incision being carried well into healthy tissue. Suturing the kidney wound did not suffice to check the bleeding, as the sutures were prone to cut through the tissue. The organ was fixed in the abdominal wound, and the hremostasis effected by means of iodoform gauze tamponade. The wound healed rapidly, but the general condition of the patient did not greatly improre. Soon after the operation the hamaturia ceased, though the nrine continued cloudy and albuminous. At the end of three weeks. the patient returned to his home, the wound having healed. Six weeks later the patient returned in a wretched condition. He complained of intolerable vesical irritability. The urine was deeply stained with blood, 
and sometimes he passed pure blood. The fuid was mingled with larger and smaller tumor masses. The examination of this material, which could also be removed from the bladder with the spoon catheter, gave the diagnosis of carcinoma of the bladder. Cystoscopic examination could not be made because of the hamorrhage, nor was it necessary.

After opening the bladder above the pubis, it was found to contain a partially disintegrated tumor the size of the fist, which was connected to the posterior wall of the bladder by a pedicle not much thicker than the thumb.. This was cut away from the mucous membrane and muscular layer by an elliptical incision around its base, and the wound in the bladder united by a few deep sutures. The opening into the bladder was closed, after a permanent catheter had been introduced. At the end of fourteen days the skin wound was found healed, the catheter was removed, and the fistula healed by granulation. After that time the patient had no more hematuria. The amount of albumin in the urine increased: and after a short period of general improvement he began to sink, and presented all the signs of nephritis. Ten weeks after the operation he died of pneumonia complicated with empyema.

At the autopsy it was found that the bladder wound was united and that there was no sign of recurrence or infiltration. The kidney which had been operated upon was firmly adherent to the capsule and surrounding tissue, but there was scarcely a trace of the operation to be found. 'The whole organ was the seat of an interstitial nephritis. The same condition was found in the piece of kidney which had been resected at the operation ; instead of being a malignant new-growth, it was interstitial nephritis.

The third case reported was one of echinococcus of the kidney. 'l'he patient was a woman, aged thirty-four years, who had been well nntil her sixteenth year, when she began to suffer from simple anzmia. She was married at the age of nineteen, and had three normal labors. The pregnancies in no way influenced her strength, though she was never able to do heavy house work. At the age of thirty-two years 
she began to grow much weaker, and since that time was confined to the bed much of the day. At that time a physician discovered a swelling of the right kidney. The right leg became swollen to such a degree as to interfere with locomotion. A few months before the operation she developed severe pain in the right side and in the abdomen, which kept her in bed. Examination showed the right kidney movable, and easily felt throngh the emaciated abdoninal walls; it was considerably enlarged, smooth and of normal shape and resistance, very tender on pressure; the left kidney was apparently normal; the spleen seemed somewhat enlarged; liver normal; urine clear and containing no pathological elements; in the middle of the right leg, on the onter side of the tibia, was a fuctuating tmmor the size of an hen's-egg; a smaller tumor of the same sort was found in the nuscle on the outer side of the right thigh; these two last tumors were removed and found to be echinococci; it was therefore conchided that the enlargement of the kidney was due to the same parasite; the organ was exposed by an incision parallel with the free border of the ribs; the kidney was easily brought np into view, and found to be about twice its normal size : prominences or changes of color were not observed; at the mpper end was a fuctuating place: incision at this point disclosed an echinococcus bladder; the organ was then freely incised, and a cyst cavity opened containing more bladders; smaller cysts were found outside of this in the kidney sull. stance; these cysts were all excised in wedges, and the wounds sutured; as in the two previous cases, to control bleeding, the organ laad to be fastened to the abdominal wound and tamponed with iodoform ganze; about a half of the kidney was removed; the pelvis remained intact. The patient made a good recovery.

Czerny, Bardenheuer, Socin and others have reported cases of resection of portions of the kidney, instead of removing the whole organ, for abscesses, horseshoe kidney and benign tumors.

Jadies P. Warbasse. 